\title{
The LUNA experiment: past and future
}

\author{
Carlo Broggini ${ }^{1, \star}$ \\ ${ }^{1}$ INFN-Padova, via F.Marzolo 8, I-35131 Padova
}

\begin{abstract}
The essential ingredients of nuclear astrophysics are the thermonuclear reactions which shape the life and death of stars and which are responsible for the synthesis of the chemical elements in the Universe. Deep underground in the Gran Sasso Laboratory the cross sections of the key reactions responsible for the hydrogen burning in stars have been measured with two accelerators of 50 and $400 \mathrm{kV}$ voltage right down to the energies of astrophysical interest. In particular, the main results obtained during the 'solar' phase of LUNA are here reviewed and their influence on our understanding of the properties of the neutrino and of the Sun is discussed. Then, the future of LUNA during the next decade is outlined. It will be mainly focused on the study of the nuclear burning stages after hydrogen burning: helium and carbon burning. All this will be accomplished thanks to a new 3.5 MV accelerator able to deliver high current beams of proton, helium and carbon which will start running under Gran Sasso in 2019.
\end{abstract}

\section{Introduction}

Stars are not perfect and eternal bodies as believed by the ancient philosophers. On the contrary, gravity triggers the birth of a star which then evolves as a fusion reactor to finally die out in a quiet or violent way, depending on its initial mass. Only hydrogen, helium and lithium are synthesized in the first minutes after the big-bang. Almost all the other elements in the Universe are produced in the reactions taking place inside the 'cauldrons' active in the cosmos, i.e. the stars. Nuclear astrophysics studies the reactions which transmute the chemical elements and, by doing so, supply stars with the power to shine. The key point of a nuclear reaction is the value of its cross section at the energy at which the reaction takes place.

In particular, the reaction occurs in the hot plasma of a star, with temperatures in the range of tens to hundreds of millions degrees, corresponding to an energy window, the Gamow peak, which is far below the Coulomb energy arising from the repulsion between nuclei. At the Gamow peak the cross section can be approximated as:

$$
\sigma(E)=\frac{S(E)}{E} \exp (-2 \pi \eta)
$$

where $S(E)$ is the astrophysical factor (which contains the nuclear physics information) and $\eta$ is given by $2 \pi \eta=31.29 Z_{1} Z_{2}(\mu / E)^{1 / 2} . Z_{1}$ and $Z_{2}$ are the nuclear charges of the interacting particles, $\mu$ is the reduced mass (in units of amu), and $E$ is the center of mass energy (in units of keV).

The extremely low value of the cross section at the stellar energies, ranging from pico to femtobarn and even below, has always prevented its measurement in a laboratory at the Earth's surface,

\footnotetext{
^e-mail: broggini@pd.infn.it, for the LUNA Collaboration
} 
where the signal to background ratio is too small mainly because of cosmic ray interactions. In order to explore the low energy domain of nuclear astrophysics LUNA (Laboratory for Underground Nuclear Astrophysics) started its activity in 1991 as a pilot project with a $50 \mathrm{kV}$ electrostatic accelerator installed inside the laboratory under Gran Sasso (Fig.1), to reproduce in the laboratory what Nature makes inside the stars (for exhaustive reviews refer to [1-3]).

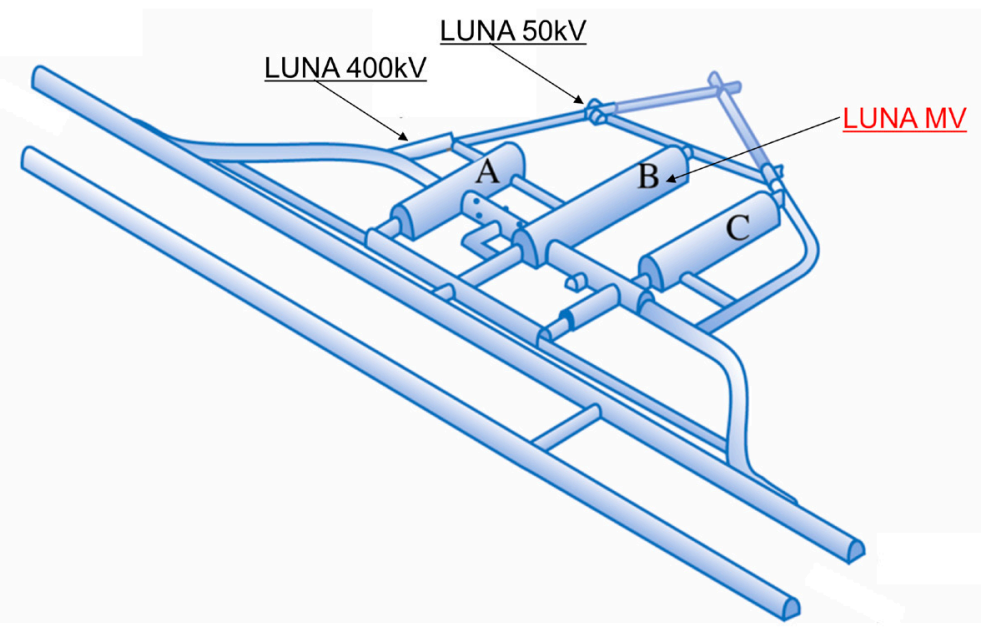

Figure 1. The underground Gran Sasso Laboratory, with the location of the three different LUNA accelerators.

\section{Background and accelerators under Gran Sasso}

The dolomite rock of Gran Sasso provides a natural shield equivalent to at least 3800 meters of water which reduces the muon and neutron fluxes by a factor $10^{6}$ and $10^{3}$, respectively. The remaining neutron flux is mainly due to $(\alpha, \mathrm{n})$ reactions in the rock, with the alpha particles coming from the ${ }^{238} \mathrm{U}$ and ${ }^{232} \mathrm{Th}$ decay chains. As a consequence, both the prompt and delayed activity from muon and neutron interactions are strongly suppressed. This way the $\gamma$-rays due the ${ }^{238} \mathrm{U}$ and ${ }^{232} \mathrm{Th}$ chains and ${ }^{40} \mathrm{~K}$ in the rock can be removed by a suitable passive shield surrounding the target and the detectors (usually low-background lead and copper), without having the shield itself activated because of cosmic ray interactions. Finally, the activity due to Radon emanating from the rock is suppressed down to the level of few tens of Bequerel $/ \mathrm{m}^{3}$ thanks to frequent air volume exchanges (once every 3.5 hours). Eventually, the background spectrum of a 'clean' $\gamma$-ray detector can be reduced by 4-5 orders of magnitude underground as compared to the overground. Not so large is the background suppression exhibited by alpha particle detectors running underground, about a factor of 15 , but still crucial for some measurements.

The accelerators used at LUNA are characterized by: high current, in the range of several hundreds $\mu \mathrm{A}$, mandatory because of the small value of the cross section to be measured, acceleration potential stable to $\leq 1 \mathrm{kV}$ over many hours in order to avoid changes in the yield due to the Coulomb barrier, and to $\leq 0.1 \mathrm{kV}$ over one hour, in order to reliably perform energy scans of targets, with the beam energy spread on a similar level $(\leq 1 \mathrm{keV})$. Two ion accelerators have hitherto been used at LUNA, and a third one will be installed soon. All three are of the electrostatic type, and the high voltage is supplied 
by Cockroft-Walton generators. The first accelerator, the LUNA $50 \mathrm{kV}$ machine, was operated with a duo-plasmatron ion source for intense and well focused hydrogen and helium beams. The main problem of this source is the relatively short lifetime. In order to solve this issue, for the LUNA 400 $\mathrm{kV}$ accelerator, in operation since 2001, a radio-frequency ion source is used to provide hydrogen or helium ions. The whole accelerator is enclosed in a pressure tank filled with 20 bar of a gas mixture consisting of nitrogen and carbon dioxide.

The third LUNA accelerator, LUNA MV, will be installed at the end of this year. Briefly, the acceleration potential of 3.5 MV is an order of magnitude larger than that of the LUNA 400 machine. The new accelerator will be able to provide not only intense ${ }^{1} \mathrm{H}^{+}$and ${ }^{4} \mathrm{He}^{+}$beams, but also carbon beams (single and double-charged, with particle current of the order of 100 and $50 \mu \mathrm{A}$, respectively). After the accelerator, the ion beam passes through an electromagnet used as a beam analyzer. The LUNA MV accelerator, as the LUNA $400 \mathrm{kV}$ one, will be equipped with two different beam lines, only one of them fed at a time. The accelerator room, with a surface of about $300 \mathrm{~m}^{2}$, will have 80 $\mathrm{cm}$ thick concrete walls and ceiling working as neutron shielding. The maximum neutron flux just outside the concrete walls will be significantly smaller than the natural neutron background at LNGS, with a similar energy spectrum.

\section{The solar phase}

The measurement of the ${ }^{3} \mathrm{He}\left({ }^{3} \mathrm{He}, 2 \mathrm{p}\right){ }^{4} \mathrm{He}$ cross section within the solar Gamow peak (16-27 keV) has been the reason why LUNA was born in 1991. Such a reaction is a key one of the hydrogen burning proton-proton chain, which is responsible for more than $99 \%$ of the solar luminosity. A resonance in its cross section at the thermal energy of the Sun was suggested at the beginning of the seventies to explain the low measured solar neutrino flux, as compared to the Standard Solar Model predictions.

The final experimental set-up for the study of ${ }^{3} \mathrm{He}\left({ }^{3} \mathrm{He}, 2 \mathrm{p}\right){ }^{4} \mathrm{He}$ was made of eight $1 \mathrm{~mm}$ thick silicon detectors of $5 \times 5 \mathrm{~cm}^{2}$ area placed around the beam inside the windowless target chamber filled with ${ }^{3} \mathrm{He}$ at the pressure of 0.5 mbar. The simultaneous detection of two protons has been the signature which unambiguously identified the ${ }^{3} \mathrm{He}\left({ }^{3} \mathrm{He}, 2 \mathrm{p}\right){ }^{4} \mathrm{He}$ fusion reaction. For the first time a nuclear reaction has been measured in the laboratory at the energy occurring in a star [4]. In particular, at the lowest energy of $16.5 \mathrm{keV}$ the cross section is $0.02 \mathrm{pb}$, which corresponds to a rate of about 2 events/month, rather low even for the "silent" experiments of underground physics. No narrow resonance has been found and, as a consequence, the astrophysical solution of the ${ }^{8} \mathrm{~B}$ and ${ }^{7} \mathrm{Be}$ solar neutrino problem based on its existence was definitely ruled out.

${ }^{3} \mathrm{He}(\alpha, \gamma){ }^{7} \mathrm{Be}$, the competing reaction for ${ }^{3} \mathrm{He}$ burning, has also been measured by LUNA both by detecting the prompt $\gamma$ rays and by counting the ${ }^{7}$ Be nuclei from their decay. The two different methods gave the same result within the total error of $4 \%$ [5].

The third cornerstone provided by LUNA to the Standard Solar Model has been the cross section of ${ }^{14} \mathrm{~N}(\mathrm{p}, \gamma){ }^{15} \mathrm{O}$ at low energy. ${ }^{14} \mathrm{~N}(\mathrm{p}, \gamma){ }^{15} \mathrm{O}$ is the slowest reaction of the first CNO cycle, it rules its energy production rate and it determines the ${ }^{13} \mathrm{~N}$ and ${ }^{15} \mathrm{O}$ solar neutrino flux, which depends almost linearly on the ${ }^{14} \mathrm{~N}(\mathrm{p}, \gamma){ }^{15} \mathrm{O}$ cross section. The results obtained by LUNA first with a germanium detector [6] and then with a high efficiency BGO set-up [7] were about a factor of two lower than the existing extrapolation from previous data. On the other hand, they were in good agreement with the results obtained with indirect methods [8]. Because of this reduction the predicted CNO neutrino yield in the Sun was decreased by about a factor of two and the lower limit on the age of the Universe inferred from the age of the oldest stellar populations, the globular clusters, was increased by $0.7-1$ billion years [9] up to 14 billion years.

We point out that the total error on the extrapolated $\mathrm{S}$-factor has been reduced by LUNA to $8 \%$ (when we started it was 50\%): $S_{1,14}(0)=1.57 \pm 0.13 \mathrm{keVb}$ [10]. Such an error is small enough to 
allow for the extraction of the carbon and nitrogen content of the Sun core from the forthcoming measurement of the CNO solar neutrinos [11]. This way it will be possible to test whether the early Sun was chemically homogeneous, a key assumption of the Standard Solar Model.

\section{The first 5 years of LUNA MV}

With the new 3.5 MV accelerator, planned to start running underground in Hall B next year (Fig.2), LUNA will enter a new phase, going to last more than 10 years. Here we describe the scientific program related only to the first 5 years of activity as given in the LUNA MV proposal [12]. The first

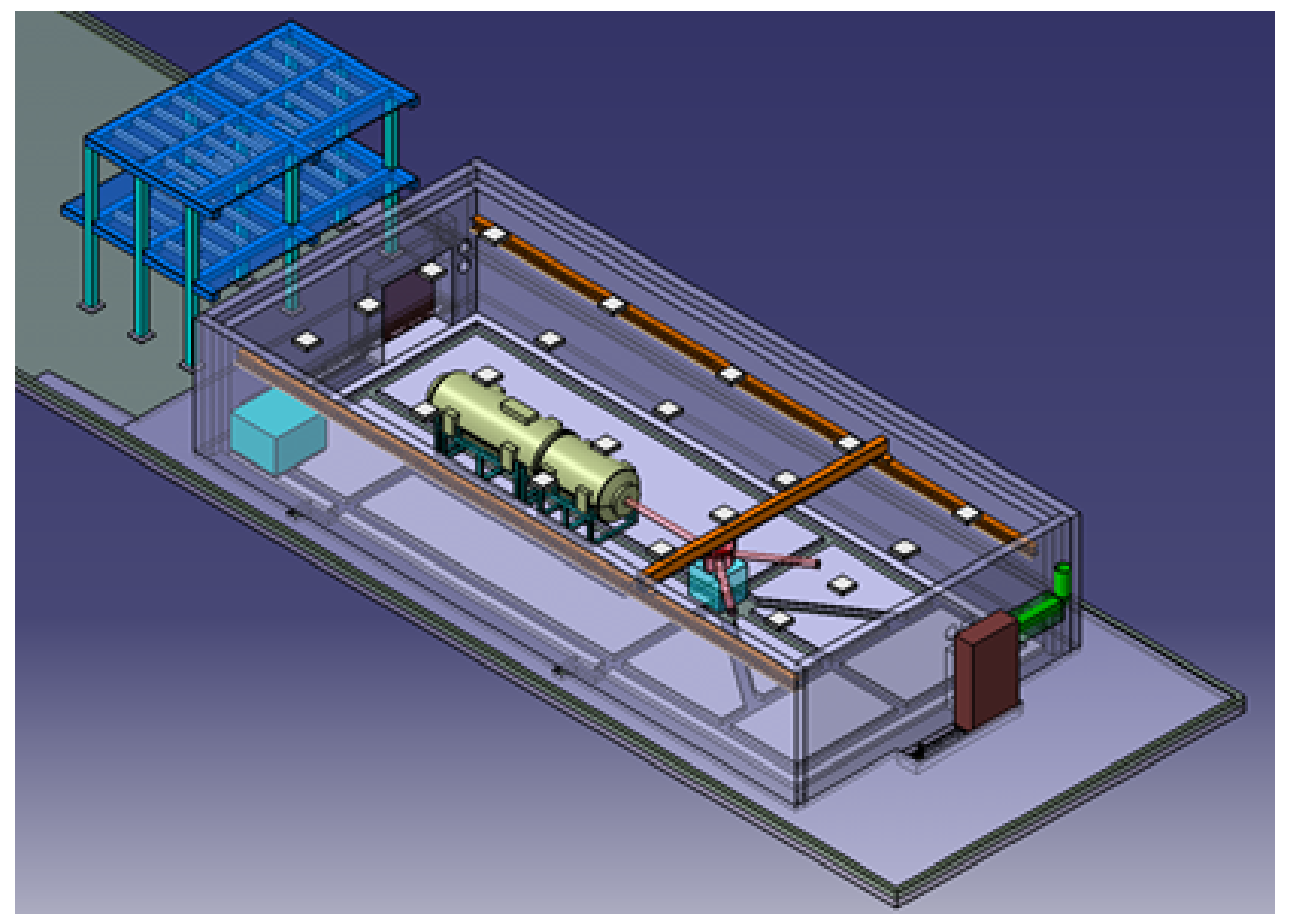

Figure 2. The LUNA MV experimental hall in the North side of Gran Sasso Hall B. The structure to the left will host counting room and ancillary facilities.

reaction to be studied will be ${ }^{14} \mathrm{~N}(\mathrm{p}, \gamma){ }^{15} \mathrm{O}$, already measured with the $400 \mathrm{kV}$ accelerator. This way we will perform the tuning of LUNA MV verifying the performances of the accelerator and of the experimental setup. In addition, a measurement over a wide energy region can provide valuable data to reduce the $8 \%$ error in the low energy extrapolation of the cross section. Such a measurement is particularly required now since a new result, obtained by using a $1 \mathrm{MV}$ and a $4 \mathrm{MV}$ accelerators at the University of Notre Dame, reported some inconsistencies for the ground state transition. Then, we will focus the activity of one of the two beam lines on the study of ${ }^{12} \mathrm{C}+{ }^{12} \mathrm{C}$ : this measure will be the flagship of the first 5 years of LUNA MV. Alternating in time with ${ }^{12} \mathrm{C}+{ }^{12} \mathrm{C}$, the study of ${ }^{13} \mathrm{C}(\alpha, n){ }^{16} \mathrm{O}$ and ${ }^{22} \mathrm{Ne}(\alpha, \mathrm{n})^{25} \mathrm{Mg}$, the two reactions responsible for neutron generation in stars, will be performed on the other beam line. 


\section{1 ${ }^{12} \mathrm{C}+{ }^{12} \mathrm{C}$}

The end of helium burning marks a branching point in stellar evolution. Lower mass stars will become stable, electron-degenerate white dwarfs, while higher mass stars will enter the quiescent carbon burning phase of their evolution. This mass limit, $\sim 9 \mathrm{M}_{\odot}$, which separates the progenitors of white dwarfs, novae and type Ia supernovae, from those of core-collapse supernovae, neutron stars, and stellar mass black holes, is given by the ${ }^{12} \mathrm{C}+{ }^{12} \mathrm{C}$ reaction rate in the temperatures range between 0.5 and $1 \mathrm{GK}$ (center of mass energies between 0.9 and $3.4 \mathrm{MeV}$ ). ${ }^{12} \mathrm{C}+{ }^{12} \mathrm{C}$ is also affecting nucleosynthesis in massive stars. The ${ }^{12} \mathrm{C}+{ }^{12} \mathrm{C}$ reaction, characterized by a Coulomb barrier of about $6.7 \mathrm{MeV}$, mainly proceeds through two different channels corresponding to the emission of a proton or an $\alpha$ particle. The Q-value for proton emission is $2.24 \mathrm{MeV}$ while that for $\alpha$ emission is $4.62 \mathrm{MeV}$. The proton and alpha channels can be measured either by detecting the charged particles or by revealing the gamma decay of the first excited state to the ground state of the ${ }^{23} \mathrm{Na}(440 \mathrm{keV})$ or ${ }^{20} \mathrm{Ne}(1634 \mathrm{keV})$ residual nuclei, respectively. Approximately, the decay of the first excited state to the ground state accounts for $50 \%$ of the total cross section.

So far, many different experiments attempted to measure the ${ }^{12} \mathrm{C}+{ }^{12} \mathrm{C}$ cross section ([12] and references therein). The lowest energy reached in a direct experiment is $2.1 \mathrm{MeV}$, with hydrogen and deuterium contaminations inside the carbon target responsible for the most severe beam induced background. In LUNA MV a detection system made of a high efficiency and ultra low intrinsic background HPGe detector complemented with silicon detectors (or telescopes) at backward angles may allow the measurement of both the $\gamma$-rays and the particles (protons and alphas). The deep underground location of the accelerator and its capability of producing an intense carbon beam offer a unique opportunity to perform such a measurement entering for the first time the low energy region below $2 \mathrm{MeV}$.

\section{The neutrons for the s-process}

The neutrons for the astrophysical s-process are supplied by two $(\alpha, \mathrm{n})$ reactions: At low temperatures, $\sim 90 \mathrm{MK},{ }^{13} \mathrm{C}(\alpha, \mathrm{n}){ }^{16} \mathrm{O}$ plays the major role. It operates in the He-rich shell of low-mass (less than 4 solar masses) AGB stars, the so called intershell, between the helium and hydrogen burning shells. It is the neutron source reaction that allows the creation of the majority of the s-process elements. At higher temperatures, $\sim 300 \mathrm{MK},{ }^{22} \mathrm{Ne}$ from one or two helium capture processes on ${ }^{18} \mathrm{O}$ or ${ }^{14} \mathrm{~N}$ is the seed for the ${ }^{22} \mathrm{Ne}(\alpha, \mathrm{n})^{25} \mathrm{Mg}$ reaction. ${ }^{22} \mathrm{Ne}(\alpha, \mathrm{n})^{25} \mathrm{Mg}$ operates in the He-burning shell of high-mass (more than 4 solar masses) AGB stars and during the core-He burning and the shell-C burning of massive stars (more than 10 solar masses). The latter is responsible for the synthesis of the s-process elements with mass number A smaller than 90.

The accurate and precise (at the level of $10 \%)$ knowledge of the ${ }^{13} \mathrm{C}(\alpha, \mathrm{n}){ }^{16} \mathrm{O}$ reaction is required at stellar temperatures in the range from 80 to 250 million $\mathrm{K}$ (below about $230 \mathrm{keV}$ center of mass energy). The current experimental situation does not fulfil such a requirement (see [12] and references therein). In particular, there exist no data from direct measurements close to the energy of astrophysical interest, the lowest energy data are affected by uncertainties that are too large to constrain extrapolations and, finally, discrepancies exist between different data sets both in energy dependence and absolute values. LUNA will study ${ }^{13} \mathrm{C}(\alpha, \mathrm{n}){ }^{16} \mathrm{O}, \mathrm{Q}=2.216 \mathrm{MeV}$, in direct kinematics both with the $400 \mathrm{kV}$ accelerator and then with the $3.5 \mathrm{MV}$ one. This way it will be possible to cover a wide energy range, to address the issue of normalization discrepancies and to minimize overall statistical and systematic uncertainties, taking advantage of the 3 orders of magnitude suppression of the laboratory neutron background. Given the low background, it may be possible to approach center of mass energies in the $200 \mathrm{keV}$ range. Finally, we point out that the same energy range of interest can be 
covered in inverse kinematics using a ${ }^{13} \mathrm{C}$ beam with energies $E=0.9-4.5 \mathrm{MeV}$. A ${ }^{4} \mathrm{He}$ gas target would be one possible solution, significantly changing the systematic uncertainties and background sources as compared to the direct kinematics measurement.

The current knowledge of the ${ }^{22} \mathrm{Ne}(\alpha, \mathrm{n})^{25} \mathrm{Mg}$ reaction is also incomplete and imprecise ([12] and references therein). Current estimates of the rate are mostly based on experimental evaluations of the dominant resonance at $832 \mathrm{keV}$ and provide the rate with an uncertainty of 20-30\%, while less than $5 \%$ is required for accurate model predictions. Furthermore, extrapolations to low energies may be affected by the unknown influence of low-energy resonances just below the neutron threshold, casting doubts on the accuracy of the values currently adopted in the stellar models. In LUNA MV it will be possible to study ${ }^{22} \mathrm{Ne}(\alpha, \mathrm{n}){ }^{25} \mathrm{Mg}(\mathrm{Q}=-478 \mathrm{keV})$ with the $\alpha$ beam on a windowless gas target of enriched ${ }^{22} \mathrm{Ne}$ surrounded by a neutron detector. It may be feasible to have an accurate cross section measurement down to the center of mass energy in the $600 \mathrm{keV}$ range. At lower energies it will be possible to exclude any resonance able to give a significant contribution to neutron production in AGB stars.

\section{Conclusions}

In 1991 LUNA started underground nuclear astrophysics in the core of Gran Sasso, below 1400 meters of dolomite rock. The extremely low background has allowed for nuclear physics experiments with very small count rate, down to a few events per year. The important reactions responsible for the hydrogen burning in the Sun have been studied for the first time down to the relevant energies, providing fundamental contributions for the prediction of the solar neutrino spectrum. Next year LUNA will enter a new phase: the study of helium and carbon burning with a 3.5 MV accelerator able to deliver high current beams of hydrogen, helium and carbon. The program of the first five years of running time is now supposed to be focused on the study of ${ }^{12} \mathrm{C}+{ }^{12} \mathrm{C}$, the reaction which is switching on carbon burning. The other two key reactions which will be studied during this phase are ${ }^{13} \mathrm{C}(\alpha, \mathrm{n}){ }^{16} \mathrm{O}$ and ${ }^{22} \mathrm{Ne}(\alpha, \mathrm{n})^{25} \mathrm{Mg}$, responsible for generating free neutrons inside stars. These neutrons give then rise to the s-process responsible for the synthesis of about half of the chemical elements beyond the iron peak in the Universe.

\section{References}

[1] H.Costantini, A.Formicola, G.Imbriani, M.Junker, C.Rolfs and F.Strieder, Rep. Progr. Phys. 72, 086301 (2009)

[2] C.Broggini, D.Bemmerer, A.Guglielmetti and R.Menegazzo, Ann. Rev. Nucl. Part. Science 60, $53(2010)$

[3] C.Broggini, D.Bemmerer, A.Caciolli and D.Trezzi, Progr. Part. Nucl. Phys. 98, 55 (2018)

[4] R.Bonetti et al., Phys. Rev. Lett. 82, 5205 (1999)

[5] F.Confortola et al., Phys. Rev. C 75, 065803 (2007)

[6] A.Formicola et al., Phys. Lett. B 591, 61 (2004)

[7] A.Lemut et al., Phys. Lett. B 634, 483 (2006)

[8] A.Mukhamedzhanov et al., Phys. Rev. C 67, 065804 (2003)

[9] G. Imbriani et al., Astron. Astrophys. 420, 625 (2004)

[10] M.Marta et al., Phys. Rev. C 78, 022802 (2008)

[11] A.Serenelli, Eur. Phys. J. A 52, 78 (2016)

[12] LUNA MV Proposal, INFN-17-19/LNGS (2017) in http://www.lnf.infn.it/sis/preprint/search.php 